\title{
The Relationship Between Sleep and Cognitive and Physical Health
}

\author{
Catherine S. Nemeskal, Jennifer Watrous, Lauren Raine, Charles Hillman \\ Danvers High School, 60 Cabot Street, Danvers, Massachusetts, 01923, USA; csnemeskal@gmail.com
}

\begin{abstract}
The relationships between sleep and aerobic fitness, intelligence, and cognitive abilities in preadolescent children was investigated in this research. Data from 483 children who were 8 - 9 years old were used to conduct correlational analyses. One parent of each participant completed a questionnaire pertaining to the typical amount of sleep received by the child participant. Each participant completed a maximal oxygen consumption test, a dual energy x-ray absorptiometry (DXA) scan, an Intelligence Test, achievement testing in reading, math, and spelling, and a modified flanker task to assess attentional inhibition. Greater amounts of sleep correlated with aerobic fitness $(r=0.15, p \leq 0.001)$. Sleep correlated with body composition, measured via body mass index $(B M I)(r=-0.15, p \leq 0.001)$ and visceral adipose tissue (VAT) $(r=-0.12, p=0.02)$. Sleep also correlated with crystallized intelligence $(r=0.18, p \leq 0.001)$ and fluid intelligence $(r=0.16, p \leq 0.001)$, as well as performance on reading $(r=0.17$, $\mathrm{p} \leq 0.001)$ and math $(\mathrm{r}=0.16, \mathrm{p}=0.01)$ achievement tests. Spelling did not correlate with sleep $(\mathrm{r}=0.09, \mathrm{p}>0.05)$. Greater amounts of sleep correlated with congruent flanker task accuracy $(\mathrm{r}=0.11, \mathrm{p}=0.03)$ and reaction time $(\mathrm{r}=0.13, \mathrm{p}=0.01)$. Finally, sleep correlated with incongruent flanker task accuracy $(r=0.16, p \leq 0.001)$ and reaction time $(r=0.13, p=0.01)$. These findings indicate a beneficial relationship of sleep with physical and cognitive health in children.

KEYWORDS: Behavioral and Social Sciences; Cognitive Psychology; Intelligence; BMI; VO2 Max; Sleep; Attentional Inhibition.
\end{abstract}

\section{- Introduction}

Human beings spend approximately one-third of their lifetime asleep. Many processes that are necessary for survival occur during sleep cycles: nerve cells communicate and build connections; the body repairs cells, tissues, and muscles; and hormones are produced throughout the body. ${ }^{1}$ But how does the time we spend asleep impact the time we spend awake? Children, 8 and 9 years old, are recommended to get 9-11 hours of sleep per night for adequate health and safety. ${ }^{2}$ Adequate sleep is particularly important for children since insufficient sleep is associated with atypical physical and cognitive development. ${ }^{3}$ According to the National Sleep Foundation, sleeping for under 7 hours or over 12 hours nightly can compromise health and development in preadolescent children and may lead to serious health problems. ${ }^{2}$ There is growing evidence linking sleep and cardiometabolic risk factors in children, particularly in terms of adiposity. ${ }^{4}$ Existing research suggests that more chronic sleep leads to a lower body mass index (BMI) in adolescents, young adults, and older adults. ${ }^{5}$ While this research exists pertaining to sleep and its effects on BMI in adolescents and adults, little is known about the effects of sleep on BMI in preadolescent children. In addition, one study also suggests that sleep influences physical activity, such that unhealthy sleep patterns are related to lower levels of cardiorespiratory fitness. ${ }^{6}$ This may be because exercise has been shown to improve sleep architecture and sleep continuity in children. ${ }^{7}$ Furthermore, research suggests that increased sleep correlates with better academic performance in adolescents. ${ }^{8}$ However, less is known about the effects of sleep on academic performance in preadolescents, as well as its effects on intelligence, both in terms of crystallized and fluid intelligence. Crystallized intelligence refers to the ability to acquire facts and knowledge. Fluid intelligence refers to the ability to think critically and use reasoning to recognize patterns. Crystallized and fluid intelligence present differently in children, as fluid intelligence increases until adolescence and then declines, whereas crystallized intelligence increases consistently. ${ }^{9}$ Additionally, research from the US Library of Medicine suggests that sleep deprivation impairs attention and inhibition in adult males. ${ }^{10}$ Attentional inhibition refers to the ability to suppress irrelevant information in order to concentrate on more pertinent information in the environment. Little is known about the relationship between sleep and attentional inhibition in children. This investigation aimed to assess the relationship between chronic sleep and cognitive and physical health in preadolescent children ages 8 to 9 years. The hypothesis is that hours of sleep received per night will correlate positively with aerobic fitness levels, crystallized and fluid intelligence, and attentional inhibition abilities, as well as correlate negatively with BMI and fat mass (i.e., visceral adipose tissue (VAT)).

\section{Methods}

A total of 483 children ages 8-9 years old were recruited to participate in this study, of which a subsample $(n=401)$ was included in this exploratory analysis. Demographic information can be found in Table 1.

To collect the necessary information to understand the relationships between sleep, fitness, BMI, intelligence, and cognition, a parent of each participant completed a basic questionnaire, which included one question pertaining to chronic sleep behavior: "How much sleep does your child 
regularly?" Answers were given in multiple choice format, with options of "under 5," "5-6," "6-7," "7-8," 8-9," "9-10", and "more than 10." For the purpose of this investigation, answers were rounded down, with the assumption that participants tend to overestimate sleep to satisfy the researcher. For example, if the "8-9 hours of sleep" option was selected, the variable of " 8 " was utilized (see Figure 1). A limitation of the study was that the questionnaire's multiple choice options did not go beyond "more than 10 " hours of sleep per night, indicated with the variable of " 10 " on all figures.

Table 1: Participant Demographics.

\begin{tabular}{|l|l|}
\hline & Mean \pm SE \\
\hline $\mathrm{N}$ & 401,211 females \\
\hline Age & $8.77 \pm 0.03$ \\
\hline $\mathrm{VO}_{2} \max (\mathrm{m} / \mathrm{kg} / \mathrm{min})$ & $39.81 \pm 0.37$ \\
\hline $\mathrm{BMl}\left(\mathrm{kg} / \mathrm{m}^{2}\right)$ & $19.10 \pm 0.21$ \\
\hline $\mathrm{IQ}$ (Standard Score) & $109.67 \pm 0.66$ \\
\hline Sleep (average hrs./ night) & $8.29 \pm 0.05$ \\
\hline
\end{tabular}

Figure 1

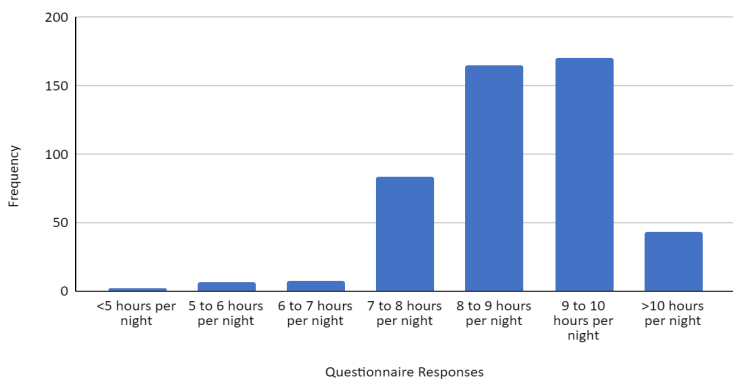

Figure 1: Average hours of sleep per night depicting the participants' responses to the question "How much sleep does your child get regularly?" The majority of participants indicate receiving 8-10 hours of sleep per night regularly.

Aerobic fitness was measured using a maximal oxygen consumption test $\left(\mathrm{VO}_{2} \max , \mathrm{ml} / \mathrm{kg} / \mathrm{min}\right) .{ }^{11}$ Participants walked or ran on a treadmill at constant speed and incline increasing until they reached volitional exhaustion. Cardiorespiratory fitness was measured as a $\mathrm{VO}_{2}$ peak. ${ }^{12}$ Participants' oxygen consumption was measured using an indirect calorimetry system (ParvoMedics TrueMax 2400). Participants walked/ran at a constant speed on a treadmill with incline increases of $2.5 \%$ every 2 minutes until volitional exhaustion. Participants wore a heart rate (HR) monitor throughout the test to calculate heart rate max. Ratings of perceived exertion (RPE) were assessed every 2 min using the children's OMNI Scale. ${ }^{13,14}$ (3) respiratory exchange ratio $(\mathrm{RER} \geq 1.0),{ }^{15}$ and/or (4) $\mathrm{RPE} \geq 8.14$ $\mathrm{VO}_{2}$ peak percentile (VO2peak\%) was then determined based on the participants' sex, age and relative score from normative data. ${ }^{16}$ Body composition was examined as BMI and VAT mass. BMI was calculated by dividing body mass $(\mathrm{kg})$ by height $(\mathrm{m})$ squared. Whole body and regional soft tissue were measured by dual energy x-ray absorptiometry (DXA) using a Hologic Discovery bone densitometer (software version 12.7.3; Hologic, Bedford, MA). VAT was estimated by using an automated algorithm that models subcutaneous abdominal adipose tissue (SAAT) at the fourth lumbar vertebra and subtracts it from the regional abdominal adipose tissue. ${ }^{17}$
See Drollette et al. ${ }^{18}$ for further details regarding fitness and body composition assessments.

Intelligence IQ was measured using scores on the Woodcock-Johnson Tests of Cognitive Abilities ${ }^{19}$ or the Kaufman Brief Intelligence Test (KBIT), ${ }^{20}$ both of which are IQ tests for children that include measures of crystallized and fluid intelligence. Each IQ test was standardized on the same scale $($ mean $=100 \pm 15)$. Academic performance was measured using the Wide Range Achievement Testing (WRAT) ${ }^{21}$ or the Kaufman Tests of Educational Abilities (KTEA), which included math, reading, and spelling. Both the WRAT and KTEA were based on a standard score with a mean of 100 and a standard deviation of 15 .

Attentional inhibition was measured in this study using a modified flanker task, which collected measures of reaction time and accuracy ${ }^{22}$ and was completed on a computer using a response pad. In the flanker task, an array of five fish appeared on a screen (see Figure 2), and the participant was asked to respond to the direction of the central (target) fish. For congruent trials, all five fish faced the same direction. For incongruent trials, the four outer fish (also referred to as flanking fish) faced in the opposite direction of the central fish, requiring the participant to use attentional inhibition to gate out the flanking fish and respond correctly to the direction of the target fish. Each participant completed 150 trials of the flanker task in two blocks of 75 trials.

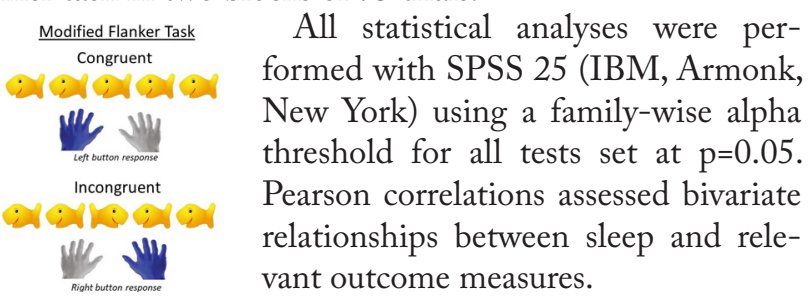

Figure 2: Example of congruent and incongruent flanker task trials.

\section{- Results and Discussion}

As seen in Table 2, a strong correlation emerged between chronic sleep and aerobic fitness (i.e., relative $\mathrm{VO}_{2}$ max; $r=0.15$; $p \leq 0.001$; see Figure 3). In addition, there were negative correlations between chronic sleep and BMI $(r=-0.15$; $\mathrm{p} \leq 0.001$; see Figure 4), as well as VAT ( $\mathrm{r}=-0.12 ; \mathrm{p}=0.02$; see Figure 5).

Table 2: Pearson Correlation Value Comparison of Measures with Chronic Sleep: As shown, nearly all relationships between sleep and cognitive and physical health measures were significant at the alpha threshold for all tests set at $\mathrm{p}=0.05$.

\begin{tabular}{|c|c|c|}
\hline & Pearson Correlation $(\mathrm{r})$ & Significance $(\mathrm{p})$ \\
\hline Relative $\mathrm{VO}_{2}$ Max & $0.15^{*}$ & $\leq 0.001$ \\
\hline Body Mass Index & $-0.15^{*}$ & $\leq 0.001$ \\
\hline Visceral Adipose Tissue & $-0.12^{*}$ & 0.02 \\
\hline Reading Scores (WRAT) & $0.17^{*}$ & $\leq 0.001$ \\
\hline Math Scores (WRAT) & $0.12^{*}$ & 0.01 \\
\hline Spelling Score (WRAT) & 0.09 & $>0.05$ \\
\hline Crystallized Intelligence (WJ), (KBIT) & $0.18^{*}$ & $\leq 0.001$ \\
\hline Fluid Intelligence (WJ), (KBIT) & $0.16^{*}$ & $\leq 0.001$ \\
\hline Congruent Reaction Time (Flanker) & $0.13^{*}$ & 0.01 \\
\hline Congruent Accuracy (Flanker) & $0.11^{*}$ & 0.03 \\
\hline Incongruent Reaction Time (Flanker) & $0.13^{*}$ & 0.01 \\
\hline Incongruent Accuracy (Flanker) & $0.16^{*}$ & $\leq 0.001$ \\
\hline
\end{tabular}




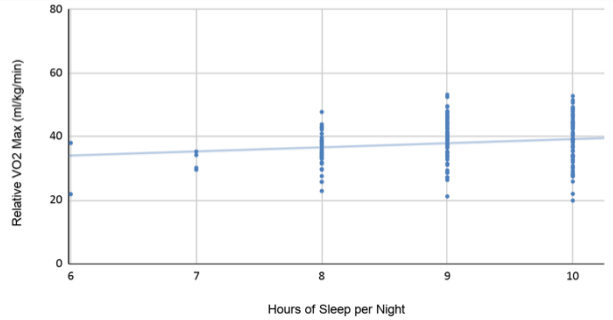

Figure 3: As shown, there is a positive correlation between average hours of sleep per night and $\mathrm{VO}_{2}$ max performance, indicating a beneficial relationship between chronic sleep and aerobic fitness.

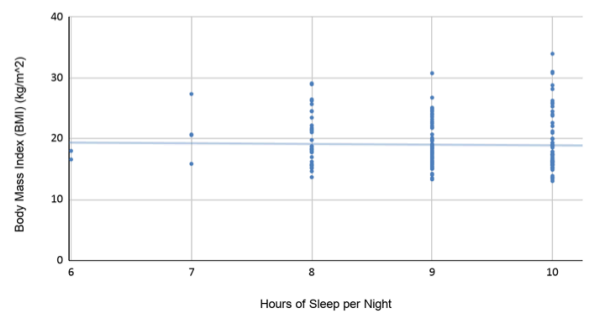

Figure 4: As shown, there is a negative correlation between average hours of sleep per night and BMI, indicating a beneficial relationship between chronic sleep and body composition.

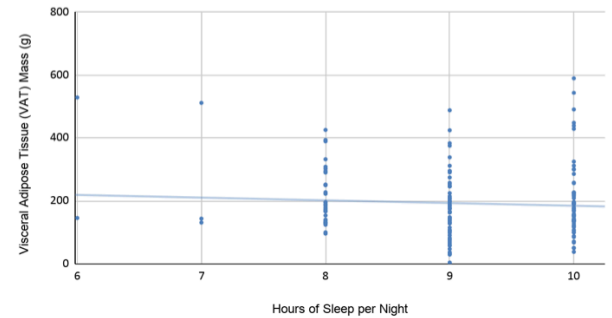

Figure 5: As shown, there is a negative correlation between average hours of sleep per night and VAT mass, indicating a beneficial relationship between chronic sleep and reduced fat mass.

Relative to the intelligence measures (see Figure 6), chronic sleep correlated with crystallized intelligence scores $(\mathrm{r}=0.18$; $\mathrm{p} \leq 0.001)$. Fluid intelligence scores also correlated with chronic sleep $(r=0.16 ; p \leq 0.001)$. Crystallized intelligence has a stronger correlation with chronic sleep, relative to fluid intelligence. For academic achievement, both reading $(r=0.17 ; \mathrm{p} \leq 0.001$; see Figure 7), and math ( $r=0.12$; $p=0.01$; see Figure 8), correlated with chronic sleep, while spelling was unrelated $(r=0.09$; p $>0.05$; see Figure 9).

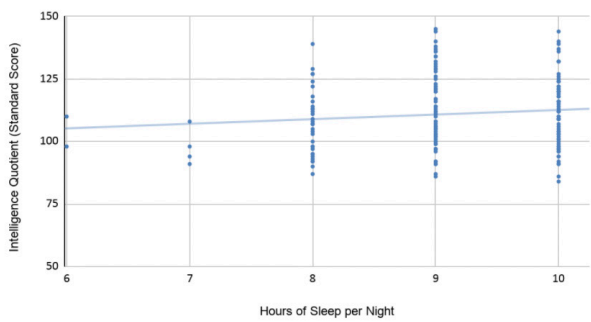

Figure 6: As shown, there is a positive correlation between average hours of sleep per night and IQ, indicating a beneficial relationship between chronic sleep and cognition.

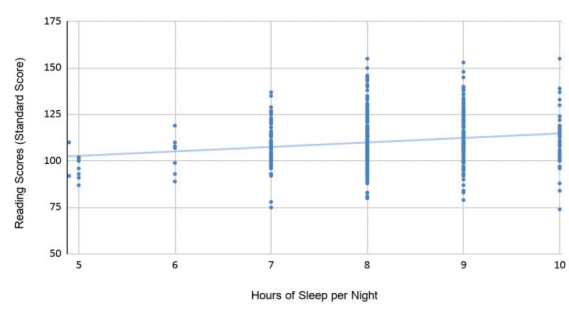

Figure 7: As shown, there is a positive correlation between average hours of sleep per night and reading scores, indicating a beneficial relationship between chronic sleep and academic achievement.

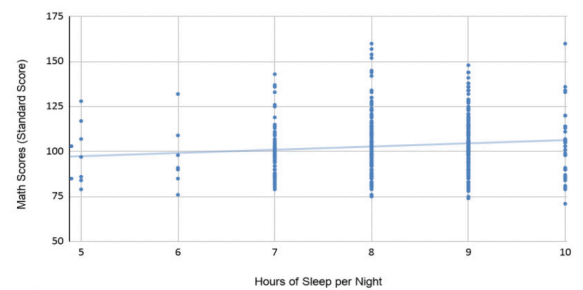

Figure 8: As shown, there is a positive correlation between average hours of sleep per night and math scores, indicating a beneficial relationship between chronic sleep and academic achievement.

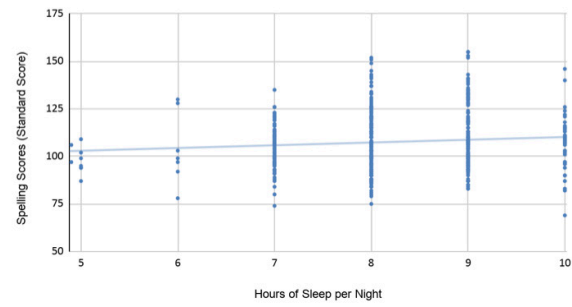

Figure 9: There is no significant correlation between average hours of sleep per night and spelling scores on an academic achievement test.

Finally, children who received more chronic sleep performed better on the flanker task. Congruent flanker trials correlated with chronic sleep, for both accuracy $(r=0.11 ; p=0.03$; see Figure 10), and reaction time ( $r=0.13 ; \mathrm{p}=0.01$; see Figure 11). Incongruent trials also correlated with sleep, for both accuracy $(r=0.16 ; p \leq 0.001$; see Figure 12), and reaction time $(r=0.13$; $\mathrm{p}=0.01$; see Figure 13 ).

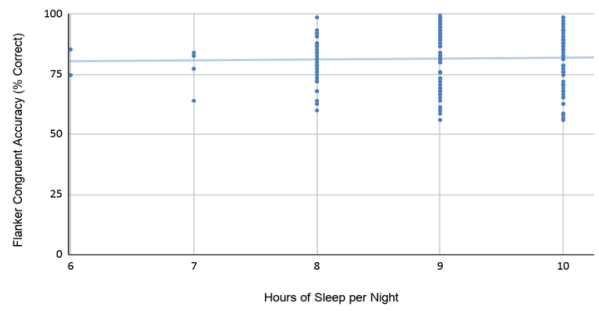

Figure 10: As shown, there is a positive correlation between average hours of sleep per night and accuracy on the congruent flanker task, indicating a beneficial relationship between chronic sleep and cognition.

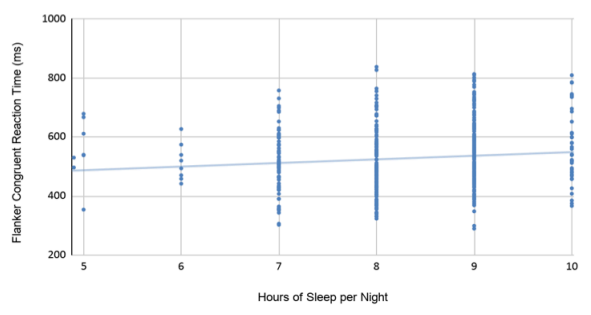

Figure 11: As shown, there is a positive correlation between average hours of sleep per night and reaction time on the congruent flanker task, indicating a beneficial relationship between chronic sleep and cognition. 


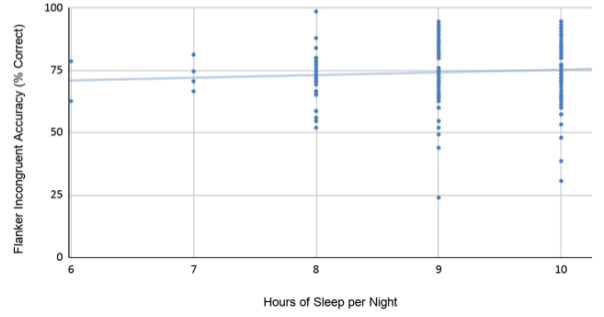

Figure 12: As shown, there is a positive correlation between average hours of sleep per night and accuracy on the incongruent flanker task, indicating a beneficial relationship between chronic sleep and cognition.

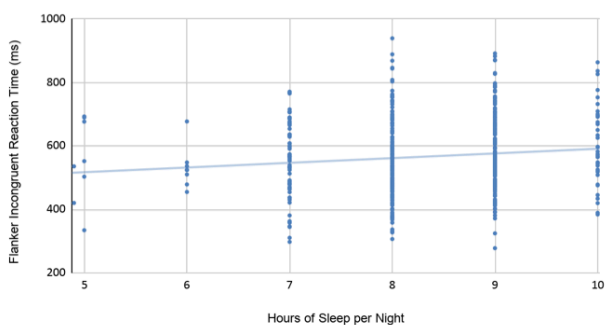

Figure 13: As shown, there is a positive correlation between average hours of sleep per night and reaction time on the incongruent flanker task, indicating a beneficial relationship between chronic sleep and cognition.

The findings demonstrate that chronically adequate amounts of sleep have a positive correlation with physical fitness, body composition, intelligence, and cognitive ability. As for physical fitness, adequate amounts of sleep correlated positively with higher levels of fitness, as demonstrated via correlations with higher $\mathrm{VO}_{2}$ max values. In addition, findings from our study highlight that chronic amounts of sleep correlated with measures of body composition, as demonstrated via correlations with low BMI, and low VAT mass.

Chronically healthy amounts of sleep correlate positively with crystallized and fluid intelligence, as well as performance on reading and math achievement tests. This suggests that chronic sleep may be beneficial for learning abilities as well as innate intelligence. In contrast, spelling was not correlated with sleep.

A relationship between chronic sleep and attentional inhibition are seen clearly in both congruent and incongruent trials of the flanker task. The correlation between sleep and performance is especially prevalent in the incongruent trials. The incongruent trials are those that require greater amounts of attentional inhibition, as the participant must actively inhibit the flanking stimuli to focus on the central (target) stimulus. The participants who slept more on a regular basis performed better on the most difficult trials. Taken together, these findings suggest that with more sleep, children may be utilizing a strategy whereby they de-emphasize their reaction time in an effort to produce more accurate responses.

Almost all correlations show a steady increase in performance from 4.9 hours of sleep to peak performance at about 8 or 9 hours of sleep per night. Thus, the data suggests that 8-9 hours of sleep may be optimal for cognitive and physical performance. There are a few potential causes for this observed trend, one of which being that the sample used in this study did not contain many participants getting 10 or more hours of sleep per night. A second limitation of the study was that on the questionnaire used for data collection, the greatest possible amount of sleep that participants could indicate was " $>10$ hours of sleep per night." Participants who provided this answer could be achieving any amount beyond 10 hours of sleep per night, including into the oversleeping range. The National Sleep Foundation identifies oversleeping as more than 12 hours of sleep per night for children ages 6-13 years, therefore some members of the "> 10 hours of sleep per night" group could be in this category of oversleeping. ${ }^{23}$ Oversleeping is implicated in type II diabetes, obesity, and depression, among other diseases and disorders. ${ }^{24}$ These external factors could be related to their decline in cognitive and physical performance.

Existing research suggests that sleep plays a positive role in the cognitive development and physical growth of infants and children. ${ }^{25}$ These findings replicate and extend findings from a recent meta-analysis and systematic review that found a significant relationship between objectively measured sleep and cognitive function, particularly for verbal IQ. ${ }^{26}$ Additionally, existing studies on sleep duration and consistent chronic sleep suggest that more sleep leads to better cognitive performance. ${ }^{27}$ These conclusions, in conjunction with our findings, suggest that the benefits of sleep are seen in various populations and can be repeated in future studies.

Based on these correlations, there is evidence that sleep is beneficial for preadolescent children in terms of both cognitive and physical health. Children who slept more on a regular basis consistently performed better on academic and cognitive tasks and demonstrated a higher level of physical and aerobic fitness than those who received fewer hours of sleep. This suggests that sleep should be prioritized in childhood to improve cognitive and physical health.

The mechanisms linking sleep to physical and cognitive health in children are poorly understood and still being investigated. However, most data links sleep restriction to an increase in energy intake, which may increase the risk of obesity. ${ }^{4}$ In terms of cognition, sleep has been shown to beneficially impact memory consolidation and learning. ${ }^{28}$ Longer sleep duration is associated with larger volume in various brain regions such as the prefrontal cortex, which is also important for cognitive function. Specifically, sleep has been shown to mediate the relationship between brain structure and cognition. ${ }^{29}$

\section{- Conclusion}

In conclusion, consistently healthy amounts of sleep have a beneficial relationship with physical (i.e., aerobic fitness, body composition) and cognitive (i.e., intelligence, attentional inhibition, and academic performance) health. Future research should extend these results using additional, more sophisticated assessments of sleep behaviors (including sleep beyond 10 hours a night), such as accelerometers, with these physical and cognitive health outcomes. Regardless, these findings provide evidence to support sleep as a predictor of childhood health and scholastic performance.

\section{Acknowledgements}

I acknowledge the support of my mentors, Jennifer Watrous, Lauren Raine, and Charles Hillman for making this project possible. 


\section{References}

1. August 14, 2020). What Happens When You Sleep? National Sleep Foundation. https://www.sleepfoundation.org/articles/ what-happens-when-you-sleep.

2. Paruthi, S., Brooks, L.J., D’Ambrosio, C., Hall, W.A., Kotagal, S., Lloyd, R.M.,Malow, B.A., Maski, K.,Nichols, C., Quan, S.F., Rosen, C.L., Troester, M.M., Wise, M.S. (2016). Recommended amount of sleep for pediatric populations: a consensus statement of the American Academy of Sleep Medicine.J Clin Sleep Med.

3. Bonuck, K., Grant, R. (2012). Sleep problems and early developmental delay: implications for early intervention programs. Intellectual and Developmental disabilities, 50(1), 41-52.

4. Quist, J.S., Sjödin, A., Chaput, J.P., Hjorth, M.F. (October 2016). Sleep and cardiometabolic risk in children and adolescents. Sleep Medicine Revierws. https://www.sciencedirect.com/science/article abs/pii/S1087079215001203.

5. Grandner, M.A., Schopfer, E.A., Sands-Lincoln, M., Jackson, N., Malhotra, A. (May 18, 2016). The Relationship between Sleep Duration and Body Mass Index Depends on Age. US National Library of Medicine. https://www.ncbi.nlm.nih.gov/pmc/articles/ PMC4700549/

6. Shang, X., Li, Y., Xu, H., Zhang, Q. Liu, A., Ma, G. (2020). The Clustering of Low Diet Quality, Low Physical Fitness, and Unhealthy Sleep Pattern and Its Association with Changes in Cardiometabolic Risk Factors in Children. Nutrients, 12(2), 591.

7. Dworak, M., Wiater, A., Alfer, D., Stephan, E., Hollmann, W., Strüder, H. K. (2008). Increased slow wave sleep and reduced stage 2 sleep in children depending on exercise intensity. Sleep medicine, 9(3), 266-272.

8. Dunster, G.P., de la Iglesia, L., Ben-Hamo, M., Nave, C., Fleischer, J.G., Panda, S., de la Iglesia, H.O. (December 12, 2018). Sleepmore in Seattle: Later school start times are associated with more sleep and better performance in high school students. Science Advances.

9. Brown, R.E. (2016). Hebb and Cattell: The Genesis of the Theory of Fluid and Crystallized Intelligence. Frontiers in Human Neuroscience. https://www.ncbi.nlm.nih.gov/pmc/ articles/PMC5156710/

10.Chern-Pin Chua, E., Fang, E., Gooley, J.J. (2017). Effects of total sleep deprivation on divided attention performance. US National Library of Medicine. https://www.ncbi.nlm.nih.gov/pmc/ articles/PMC5699793/

11.Khan, N.A., Raine, L.B., Drollette, E.S., Scudder, M.R., Pontifex, M.B., Castelli, D.M., Donovan, S.M., Evans, E.M., Hillman, C.H. (March 31, 2014). Impact of the FITKids Physical Activity Intervention on Adiposity in Prepubertal Children. Pediatrics. http://labs.kch.illinois.edu/Research/Labs/neurocognitivekinesiology/files/Articles/Khan_2014_ImpactOfTheFITKids.pdf

12.Winsley, R.J., Armstrong, N., Middlebrooke, A.R., Ramos-Ibanez, N., Williams, C.A. (2006). Aerobic fitness and visceral adipose tissue in children. Acta Paediatr. 95: 1435-1438.

13.Utter, A.C., Robertson, R.J., Nieman, D.C., Kang, J. (January 2002). Children's OMNI Scale of Perceived Exertion: walking/ running evaluation. National Library of Medicine. https://pubmed. ncbi.nlm.nih.gov/11782659/

14.Pescatello, L.S. (2014). ACSM's guidelines for exercise testing and prescription. Wolters Kluwer/Lippincott Williams \& Wilkins Health. American College of Sports Medicine. $456 \mathrm{p}$.

15.Bar-Or, O. (1983). Pediatric Sports Medicine for the Practitioner. Comprehensive Manuals in Pediatrics.

16. Shvartz, E., Reibold, R.C. (January 1990). Aerobic fitness norms for males and females aged 6 to 75 years: a review. National Library of Medicine. https://pubmed.ncbi.nlm.nih.gov/2405832/
17.Micklesfield, L.K., Goedecke, J.H., Punyanitya, M., Wilson, K.E., Kelly, T.L. (2012). Dual-Energy X-Ray Performs as Well as Clinical Computed Tomography for the Measurement of Visceral Fat. Obesity. 2012;20(5):1109-14.

18.Drollette, E.S., Pontifex, M.B., Raine, L.B., Scudder, M.R., Moore, R.D., Kao, S., Westfall, D.R., Wu, C., Kamijo, K., Castelli, D.M., Khan, N.A., Kramer, A.F., Hillman, C.H.(October 4,2017). Effects of the FITKids physical activity randomized controlled trial on conflict monitoring in youth. https://onlinelibrary.wiley.com/doi/ epdf/10.1111/psyp. 13017

19.Hasinger, E. (2020). Woodcock-Johnson Test Guide. https://www.tests.com/Woodcock-Johnson-Testing

20.Kaufman, A.S., Kaufman, N.L. Kaufman Brief Intelligence Test, Second Edition. https://www.wpspublish.com/kbit-2-

kaufman-brief-intelligence-test-second-edition

21.Wilkinson, G.S., Robertson, G.J. Wide Range

Achievement Test Fifth Edition. Pearson Assessments. https:// www.pearsonassessments.com/store/usassessments/en/Store/ Professional-Assessments/Academic-Learning/Brief/WideRange-Achievement-Test-\%7C-Fifth-Edition/p/100001954.htm

22.Eriksen, B.A., Eriksen, C.W. (1974). Effects of noise letters upon identification of a target letter in a non-search task. Perception and Psychophysics, 16, 143-149.

23.Hirshkowitz, M., Whiton, K., Albert, S.M., Alessi, C., Bruni, O., DonCarlos, L., Hazen, N., Herman, J., Katz, E.S., KheirandishGozal, L., Neubauer, D.N., O’Donnell, A.E., Ohayon, M., Peever, J., Rawding, R., Sachdeva, R.C., Setters, B., Vitiello, M.V., Ware, J.C., Hillard, P.J. (January 14, 2015). National Sleep Foundation's sleep time duration recommendations: methodology and results summary. National Sleep Foundation. https://www. sleephealthjournal.org/article/S2352-7218(15)00015-7/fulltext

24.Oversleeping: Bad for Your Health? Johns Hopkins Medicine. https://www.hopkinsmedicine.org/health/wellness-andprevention/oversleeping-bad-for-your-health

25.Tham, E.K.H., Schneider, N., Broekman, B.F.P. (May 15, 2017). Infant sleep and its relation with cognition and growth: a narrative review. US Library of Medicine. https://www.ncbi.nlm.nih.gov/ pmc/articles/PMC5440010/

26.Short, M.A., Blunden, S., Rigney, G., Matricciana, L., Coussens, S., Reynolds, C.M., Galland, B. (June 2018). Cognition and objectively measured sleep duration in children: a systematic review and meta-analysis. Sleep Health. https://www.sciencedirect.com/ science/article/abs/pii/S2352721818300135

27.Mantua, J., Simonelli, G. (March, 2019). Sleep duration and cognition: is there an ideal amount? Oxford Academic. https:// academic.oup.com/sleep/article/42/3/zsz010/5288680

28.Ashworth, A., Hill, C.M., Karmiloff-Smith, A., Dimitriou, D. (December 16, 2013). Sleep enhances memory consolidation in children. Journal of Sleep Research. https://onlinelibrary.wiley. com/doi/full/10.1111/jsr.12119

29.Cheng, W., Rolls, E., Gong, W., Du, J., Zhang, J., Zhang, X., Li, F., Feng, J. (February 3, 2020). Sleep duration, brain structure, and psychiatric and cognitive problems in children. Molecular Psychiatry. https://pubmed.ncbi.nlm.nih.gov/32015467/

\section{Author}

Catherine Nemeskal is a senior at Danvers High School in Danvers, Massachusetts. She will be attending Dartmouth College where she plans to pursue a double major in neuroscience and economics. 